\title{
Front Matter: Volume 7684
}

, "Front Matter: Volume 7684," Proc. SPIE 7684, Laser Radar Technology and Applications XV, 768401 (20 May 2010); doi: 10.1117/12.867779

SDIE Event: SPIE Defense, Security, and Sensing, 2010, Orlando, Florida, United SPIE. States 


\section{PROCEEDINGS OF SPIE}

\section{Laser Radar Technology and Applications XV}

Monte D. Turner

Gary W. Kamerman

Editors

6-9 April 2010

Orlando, Florida, United States

Sponsored and Published by

SPIE

Volume 7684

Proceedings of SPIE, 0277-786X, v. 7684 
The papers included in this volume were part of the technical conference cited on the cover and title page. Papers were selected and subject to review by the editors and conference program committee. Some conference presentations may not be available for publication. The papers published in these proceedings reflect the work and thoughts of the authors and are published herein as submitted. The publisher is not responsible for the validity of the information or for any outcomes resulting from reliance thereon.

Please use the following format to cite material from this book:

Author(s), "Title of Paper," in Laser Radar Technology and Applications XV, edited by Monte D. Turner, Gary W. Kamerman, Proceedings of SPIE Vol. 7684 (SPIE, Bellingham, WA, 2010) Article CID Number.

ISSN 0277-786X

ISBN 9780819481481

Published by

SPIE

P.O. Box 10, Bellingham, Washington 98227-0010 USA

Telephone +1 3606763290 (Pacific Time) · Fax +1 3606471445

SPIE.org

Copyright (C) 2010, Society of Photo-Optical Instrumentation Engineers

Copying of material in this book for internal or personal use, or for the internal or personal use of specific clients, beyond the fair use provisions granted by the U.S. Copyright Law is authorized by SPIE subject to payment of copying fees. The Transactional Reporting Service base fee for this volume is $\$ 18.00$ per article (or portion thereof), which should be paid directly to the Copyright Clearance Center (CCC), 222 Rosewood Drive, Danvers, MA 01923. Payment may also be made electronically through CCC Online at copyright.com. Other copying for republication, resale, advertising or promotion, or any form of systematic or multiple reproduction of any material in this book is prohibited except with permission in writing from the publisher. The CCC fee code is 0277-786X/10/\$18.00.

Printed in the United States of America.

Publication of record for individual papers is online in the SPIE Digital Library.

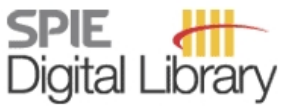

SPIEDigitalLibrary.org

Paper Numbering: Proceedings of SPIE follow an e-First publication model, with papers published first online and then in print and on CD-ROM. Papers are published as they are submitted and meet publication criteria. A unique, consistent, permanent citation identifier (CID) number is assigned to each article at the time of the first publication. Utilization of CIDs allows articles to be fully citable as soon they are published online, and connects the same identifier to all online, print, and electronic versions of the publication. SPIE uses a six-digit CID article numbering system in which:

- The first four digits correspond to the SPIE volume number.

- The last two digits indicate publication order within the volume using a Base 36 numbering system employing both numerals and letters. These two-number sets start with 00, 01, 02, 03, 04, $05,06,07,08,09,0 A, 0 B \ldots 0 Z$, followed by 10-1Z, 20-2Z, etc.

The CID number appears on each page of the manuscript. The complete citation is used on the first page, and an abbreviated version on subsequent pages. Numbers in the index correspond to the last two digits of the six-digit CID number. 


\section{Contents}

ix Conference Committee

\section{D IMAGING I}

768402 3D imaging laser radar using Geiger-mode APDs: analysis and experiments [7684-01]

Y. Guo, G. Huang, R. Shu, Shanghai Institute of Technical Physics (China)

768403 Active 3D camera design for target capture on Mars orbit [7684-03]

P. Cottin, F. Babin, D. Cantin, INO (Canada); A. Deslauriers, B. Sylvestre, Neptec Design Group Ltd. (Canada)

768404 Improved performance ladar receiver [7684-04]

B. Dion, N. Bélanger, J. Lauzon, P. Lepage, M. Tremblay, Esterline-CMC Electronics Inc. (Canada)

768405 Compact 3D flash lidar video cameras and applications [7684-05]

R. Stettner, Advanced Scientific Concepts, Inc. (United States)

768406 3D range-gated imaging in scattering environments [7684-06]

M. Laurenzis, F. Christnacher, D. Monnin, Institut Franco-Allemand de Recherches de Saint-Louis (France); I. Zielenski, Wehrtechnische Dienststelle für Waffen und Munition (Germany)

$768407 \quad 3 D-$ LZ helicopter ladar imaging system [7684-07]

J. Savage, W. Harrington, R. A. McKinley, Air Force Research Lab. (United States); H. N. Burns, H.N. Burns Engineering Corp. (United States); S. Braddom, Z. Szoboszlay, Army

Aeroflightdynamics Directorate (United States)

768408 Terrain classification of ladar data over Haitian urban environments using a lower envelope follower and adaptive gradient operator [7684-66]

A. L. Neuenschwander, The Univ. of Texas at Austin (United States); M. M. Crawford, Purdue Univ. (United States); L. A. Magruder, The Univ. of Texas at Austin (United States); C. A. Weed, MIT Lincoln Lab. (United States); R. Cannata, Harris Corp. (United States); D. Fried,

R. Knowlton, R. Heinrichs, MIT Lincoln Lab. (United States)

768409 Spectral ladar: towards active 3D multispectral imaging [7684-09]

M. A. Powers, General Dynamics Corp. (United States) and Univ. of Maryland, College Park (United States); C. C. Davis, Univ. of Maryland, College Park (United States)

7684 OA Product chain analysis of three-dimensional imaging laser radar systems employing Geiger-mode avalanche photodiodes [7684-10]

N. A. Lopez, R. R. Burton, G. W. Kamerman, B. H. Miles, W. Crouch, FastMetrix, Inc. (United States) 
7684 OB The dawn of optical radar: a story from another side of the globe (Invited Paper) [7684-1 1] V. Molebny, National Taras Shevchenko Univ. of Kyiv (Ukraine); P. Zarubin, State Unitary Enterprise V.K. Orlov Granat Development Bureau (Russian Federation); G. Kamerman, FastMetrix, Inc. (United States)

7684 OC $\quad 32 \times 32$ Geiger-mode ladar camera [7684-12] P. Yuan, R. Sudharsanan, X. Bai, J. Boisvert, P. McDonald, E. Labios, Spectrolab, Inc. (United States); M. S. Salisbury, G. M. Stuart, H. Danny, A. A. Portillo, A. B. Roybal, Boeing-SVS, Inc. (United States); S. Van Duyne, G. Pauls, S. Gaalema, Black Forest Engineering, LLC (United States)

7684 OD Intensified imaging photon-counting technology for enhanced flash lidar performance [7684-13]

C. J. Grund, A. Harwit, Ball Aerospace \& Technologies Corp. (United States)

$7684 \mathrm{OE} \quad$ Foliage penetration obscuration probability density function analysis from overhead canopy photos for gimbaled linear-mode and Geiger-mode airborne lidar [7684-14] R. R. Burton, FastMetrix, Inc. (United States)

7684 OF Calibration targets and standards for 3D lidar systems [7684-08]

B. H. Miles, G. W. Kamerman, D. K. Fronek, P. Eadon, FastMetrix, Inc. (United States)

\section{ENVIRONMENTAL MONITORING}

7684 ol Atmospheric aerosol characterization using multiwavelength multistatic light scattering [7684-17]

A. M. Wyant, The Pennsylvania State Univ. (United States); M. G. Snyder, North Carolina State Univ. (United States); L. Brouwer, Alion Science and Technology (United States);

C. R. Philbrick, The Pennsylvania State Univ. (United States) and North Carolina State Univ. (United States)

$76840 \mathrm{~J}$ Optical remote sensing techniques characterize the properties of atmospheric aerosols [7684-18]

R. Philbrick, North Carolina State Univ. (United States) and The Pennsylvania State Univ. (United States); H. Hallen, North Carolina State Univ. (United States); A. Wyant, The Pennsylvania State Univ. (United States); T. Wright, M. Snyder, North Carolina State Univ. (United States)

7684 OK Lidar, TEOM, and sunphotometer measured and model reconstructed atmospheric parameters [7684-19]

D. V. Vladutescu, The City College of The City Univ. of New York (United States) and New York City College of Technology (United States); B. Gross, Y. Wu, F. Moshary, S. Ahmed, New York City College of Technology (United States)

$7684 \mathrm{OL}$ Standoff detection of explosives in open environment using enhanced photodissociation fluorescence [7684-20]

T. Arusi-Parpar, S. Fastig, J. Shapira, B. Shwartzman, D. Rubin, Y. Ben-Hamo, A. Englander, Soreq Nuclear Research Ctr. (Israel) 
7684 ON The application of iterative closest point (ICP) registration to improve 3D terrain mapping estimates using the flash 3D ladar system [7684-22]

J. Woods, E. E. Armstrong, Air Force Research Lab. (United States); W. Armbruster, FGAN-FOM (Germany); R. Richmond, ITT Information Systems (United States)

768400 Computational experiments on super-resolution enhancement of flash lidar data [7684-23] A. Bulyshev, Analytical Mechanics Associates, Inc. (United States); G. Hines, M. Vanek, F. Amzajerdian, R. Reisee, NASA Langley Research Ctr. (United States); D. Pierrottet, Coherent Applications, Inc. (United States)

7684 OP Redundancy analysis of raw Geiger-mode laser radar data [7684-24]

N. A. Lopez, G. W. Kamerman, FastMetrix, Inc. (United States)

$7684 \mathrm{OQ}$ Scene-based algorithm for range/intensity estimation correction for the flash 3D ladar system [7684-25]

S. Jordan, Air Force Research Lab. (United States); E. Armstrong, OptiMetrics, Inc. (United States); H. Larsson, Swedish Defence Research Agency (Sweden); M. Gebhardt, Air Force Research Lab. (United States); O. Steinvall, Swedish Defence Research Agency (Sweden)

7684 OR Signal processing on waveform data from the Eyesafe Ladar Testbed (ELT) [7684-26] K. D. Neilsen, S. E. Budge, R. T. Pack, Utah State Univ. (United States)

7684 OS Automated in-track and cross-track airborne flash ladar image registration for wide-area mapping [7684-27]

C. M. Wong, J. E. Logan, C. Bracikowski, B. K. Baldauf, Northrop Grumman Aerospace Systems (United States)

\section{COHERENT SYSTEMS}

7684 OT A history of laser radar in the United States (Invited Paper) [7684-65]

P. F. McManamon, Univ. of Dayton (United States); G. Kamerman, FastMetrix, Inc. (United States); M. Huffaker, Coherent Investments (United States)

7684 OU Coherent high-resolution sparse aperture imaging testbed [7684-29]

I. Anisimov, N. J. Miller, D. Shemano, P. F. McManamon, Ladar and Optical Communications Institute (United States); J. W. Haus, Univ. of Dayton (United States)

7684 OV Compact diode laser homodyne vibrometers [7684-30]

C. J. Grund, LightWorks, LLC (United States); H. Guenther, J. Connolly, Innovative Photonic Solutions (United States)

7684 OW Coherent integration efficiency, diversity, and detectivity of temporally integrated random coherent ladar signals [7684-31]

P. Gatt, D. Jacob, Lockheed Martin Coherent Technologies (United States) 


\section{AUTOMATIC TARGET RECOGNITION LIDAR}

7684 0X Feature extraction using voxel aggregation of focused discrete lidar data [7684-33]

S. Hagstrom, D. Messinger, S. Brown, Rochester Institute of Technology (United States)

7684 OY Three-dimensional transformation for automatic target recognition using lidar data [7684-34]

R. D. Nieves, W. D. Reynolds, Jr., ITT Corp. (United States)

$7684 \mathrm{OZ}$ Laser gated viewing: An enabler for automatic target recognition? [7684-35]

E. Bovenkamp, TNO Science and Industry (Netherlands); K. Schutte, TNO Defence, Security and Safety (Netherlands)

768410 Obstruction detection comparison of small-footprint full-waveform and discrete return lidar [7684-36]

L. A. Magruder, A. L. Neuenschwander, S. P. Marmillion, The Univ. of Texas at Austin (United

States); S. A. Tweddale, Army Corps of Engineers (United States)

\section{SIMULATION AND MODELING}

$768411 \quad$ Simulating full-waveform LIDAR [7684-37]

A. M. Kim, R. C. Olsen, C. F. Borges, Naval Postgraduate School (United States)

768412 Receiver-operating characteristic for several multiple hypothesis range-rate filter algorithms [7684-38]

D. G. Youmans, SPARTA, Inc. (United States)

768413 Accuracy of multi-look geo-coding [7684-39]

E. M. Weidaw, M. W. Roth, M. Z. Brown, A. E. Scheck, The Johns Hopkins Univ. Applied Physics Lab. (United States)

768415 Physical modeling of 3D and 4D laser imaging [7684-41]

G. Anna, D. Hamoir, L. Hespel, F. Lafay, N. Rivière, B. Tanguy, ONERA (France)

\section{MARINE AND MARITIME SYSTEMS}

768416 Calibration of a seafloor microtopography laser high-definition profiler [7684-42]

N. P. Chotiros, K. R. Loeffler, T.-A. N. Nguyen, The Univ. of Texas at Austin (United States)

768417 Laser imaging of small surface vessels and people at sea [7684-44]

O. Steinvall, M. Elmqvist, K. Karlsson, H. Larsson, M. Axelsson, Swedish Defence Research Agency (Sweden)

\section{ADVANCED SYSTEMS AND COMPONENTS I}

768418 Flight test and simulation results of an integrated dual airborne laser scanner (DALS)/INS navigator [7684-45]

A. Vadlamani, M. Uijt de Haag, Ohio Univ. (United States) 
768419 From long range to high precision: pushing the limits of pulsed-time-of-flight measurement [7684-46]

M. Pfennigbauer, RIEGL Laser Measurement Systems GmbH (Austria); B. Möbius, Jena-Optronik GmbH (Germany); A. Ullrich, RIEGL Laser Measurement Systems GmbH (Austria); J. Pereira do Carmo, European Space Agency (Netherlands)

7684 1A A novel NLFM waveform generator using tunable integrated optical ring resonators: simulation and proof of concept experiment [7684-48]

D. B. Adams, W. T. Snider, C. K. Madsen, Texas A\&M Univ. (United States)

7684 IB High-power high-rep-rate SLM lasers [7684-49]

J. Kilmer, G. Ross, Y. Yin, Photonics Industries International, Inc. (United States)

7684 ID Special modulated beams for cylindrical coordinates in anisotropic media using computer algebra [7684-51]

S. Echeverri Chacón, Univ. EAFIT (Colombia)

7684 IE MEMS-scanned ladar sensor for small ground robots [7684-52]

B. L. Stann, J. F. Dammann, M. M. Giza, Army Research Lab. (United States); P.-S. Jian, Aerotek Inc. (United States); W. B. Lawler, H. M. Nguyen, L. C. Sadler, Army Research Lab. (United States)

\section{ADVANCED SYSTEMS AND COMPONENTS II}

7684 IF Improving quality of laser scanning data acquisition through calibrated amplitude and pulse deviation measurement [7684-53]

M. Pfennigbauer, A. Ullrich, RIEGL Laser Measurement Systems GmbH (Austria)

$7684 \mathrm{1H} \quad$ Fusing waveform lidar and hyperspectral data for species-level structural assessment in savanna ecosystems [7684-55]

D. Sarrazin, J. van Aardt, Rochester Institute of Technology (United States); G. P. Asner, Carnegie Institution for Science (United States); J. McGlinchy, D. W. Messinger, J. Wu, Rochester Institute of Technology (United States)

768411 An improvement on accuracy of laser radar using a Geiger-mode avalanche photodiode by time-of-flight analysis with Poisson statistics [7684-56]

M. S. Oh, H. J. Kong, KAIST (Korea, Republic of)

$76841 \mathrm{~J}$ Extended-range digital holographic imaging [7684-64]

J. C. Marron, Lockheed Martin Coherent Technologies (United States); R. L. Kendrick, Lockheed Martin Advanced Technology Ctr. (United States); S. T. Thurman,

N. L. Seldomridge, T. D. Grow, C. W. Embry, A. T. Bratcher, Lockheed Martin Coherent Technologies (United States)

\section{POSTER SESSION}

7684 IK Statistical comparison between Hysplit sounding and lidar observation of planetary boundary layer characteristics over New York City [7684-57]

C. M. Gan, The City College of New York (United States) and The Graduate Center (United States); Y. Wu, B. Gross, F. Moshary, The City College of New York (United States) 
$7684 \mathrm{IL} \quad$ Atmospheric cross-wind and turbulence measurements using turbulence-induced scintillations [7684-58]

J. Shapira, O. Porat, M. Livneh, Z. Wies, D. Heflinger, S. Fastig, Y. Glick, A. Engel, Soreq Nuclear Research Ctr. (Israel)

7684 1M Remote qualitative profiling of the cross-wind flow with lidar [7684-59]

G. Machavariani, J. Shapira, U. Talmud, O. Porat, S. Fastig, Soreq Nuclear Research Ctr. (Israel)

768410 Multispectral lidar system: design, build, and test [7684-61]

S. Fastig, Y. Ehrlich, S. Pearl, E. Naor, Y. Kraus, T. Inbar, D. Katz, Soreq Nuclear Research Ctr. (Israel)

Author Index 


\title{
Conference Committee
}

\author{
Symposium Chair
}

Michael T. Eismann, Air Force Research Laboratory (United States)

Symposium Cochair

William Jeffrey, HRL Laboratories, LLC (United States)

Conference Chairs

Monte D. Turner, Defense Advanced Research Projects Agency

(United States)

Gary W. Kamerman, FastMetrix, Inc. (United States)

Program Committee

Phillip Gatt, Lockheed Martin Coherent Technologies (United States)

James C. Lamoreux, NASA Johnson Space Center (United States)

Vasyl V. Molebny, National Taras Shevchenko University of Kyiv (Ukraine)

C. Russell Philbrick, The Pennsylvania State University (United States)

Upendra N. Singh, NASA Langley Research Center (United States)

Ove Steinvall, Swedish Defence Research Agency (Sweden)

\section{Session Chairs}

1 Introduction to CZMIL: A Multisensor Airborne Mapping System: Joint Session with Conference 7695

Paul E. Lewis, National Geospatial-Intelligence Agency (United States)

Monte D. Turner, Defense Advanced Research Projects Agency

(United States)

2 CZMIL Hardware: Joint Session with Conference 7695

Grady H. Tuell, Optech International, Inc. (United States)

3 CZMIL Algorithms and Software: Joint Session with Conference 7695

Grady H. Tuell, Optech International, Inc. (United States)

$4 \quad 3 \mathrm{D}$ Imaging I

Monte D. Turner, Defense Advanced Research Projects Agency

(United States) 
3D Imaging II

Monte D. Turner, Defense Advanced Research Projects Agency

(United States)

$6 \quad$ Environmental Monitoring

Gary W. Kamerman, FastMetrix, Inc. (United States)

7 Data Processing and Algorithms

Phillip Gatt, Lockheed Martin Coherent Technologies (United States)

8 Coherent Systems

Ove Steinvall, Swedish Defence Research Agency (Sweden)

9 Automatic Target Recognition Lidar

C. Russell Philbrick, The Pennsylvania State University (United States)

10 Simulation and Modeling

Norman A. Lopez, FastMetrix, Inc. (United States)

11 Marine and Maritime Systems

Kevin R. Slocum, National Geospatial-Intelligence Agency (United States)

12 Advanced Systems and Components I

Vasyl V. Molebny, National Taras Shevchenko University of Kyiv (Ukraine)

13 Advanced Systems and Components II

Vasyl V. Molebny, National Taras Shevchenko University of Kyiv (Ukraine) 